\title{
Family Partnerships, Palliative Care and End of Life
}

\author{
Louise Brent, Julie Santy-Tomlinson, and Karen Hertz
}

The involvement of families, friends and others important to the patient has always been central in person-centred, individualised care. Following fragility fracture, many patients wish for their family and significant others to be involved in their care, both during the hospital stay and following discharge and it is often expected that families will provide, or lead, continuing care once they are discharged.

For some patients, a significant fracture such as hip fracture may be a final element of a struggle with physical and psychological decline and frailty. It may signal the nearing of, and may hasten, the end of life. For these patients, person- and family-centred care may be needed that is focused on dignity and comfort, rather than surgical intervention. More people are living longer and living with serious, life-limiting or life-threatening conditions.

Palliative care has been associated with cancer, but has evolved to include patients with a range of complex and chronic medical conditions, and focuses on conservative management and care rather than invasive medical interventions, but is not necessarily focused on the end of life, rather on comfort, symptom management, dignity and family-centred care.

In some cases, patients with fragility fractures may become seriously ill due to their frailty and the complications of the fracture or surgery. This may result in

\footnotetext{
L. Brent

National Office of Clinical Audit, St Stephen's Green, Dublin 2, Ireland

J. Santy-Tomlinson $(\bowtie)$

Faculty of Biology, Medicine and Health, Division of Nursing, Midwifery and Social Work, School of Health Sciences, The University of Manchester, Manchester, UK

e-mail: Julie.santy-tomlinson@manchester.ac.uk

K. Hertz

Specialised Division, University Hospital of North Midlands,

Stoke-on Trent, Staffordshire, UK

e-mail: Karen.hertz@uhnm.nhs.uk
} 
sudden death but can also lead to an awareness that death is pending, but the process of dying may take several days or weeks. Patients and their families expect good end of life care that involves management of physical symptoms such as pain, breathlessness, nausea and increasing fatigue as well as the anxiety, depression and social and spiritual difficulties that can accompany the end of life. Whether the patient dies in the hospital or at home, care should be based on effective MDT working [1] that includes collaboration with the patient and their family and other informal carers.

This chapter will explore the importance of family partnerships in healthcare and approaches to palliative and end of life for care for patients with a fragility fracture.

\subsection{Learning Outcomes}

At the end of the chapter, the nurse will be able to:

- Discuss the role of family and friends as carers and how nurses should facilitate family involvement in care

- Discuss the concepts of palliative and end of life care following fragility fracture

- Be aware of medicolegal issues that affect decision-making in end of life care

- Define and practice effective palliative care

- Define and practice good end of life care

- Discuss the role of the fragility fracture practitioner in palliative and end of life care.

\subsection{Family Partnerships and Involvement in Care}

There has been significant change in the way healthcare professionals view care delivery, from a medical-centric model to a patient-centred approach. The term "patient-centred medicine" was first coined in 1969 [2], and the Picker Commonwealth Program for Patient-Centred Care (subsequently the Picker Institute) developed the term "patient-centred" in 1998 [3]. It is now acknowledged that a key priority for patients is how their own wishes and those of their families and carers are integrated throughout the care process. Healthcare workers are now expected to place an emphasis on collaborating with patients and families of all ages, at all levels of care and in all healthcare settings.

The concept of family caregiving has also developed significantly and now reflects the changing nature of "family" in society. Much informal care is given by individuals who would not traditionally be considered family members and such "informal caregiving" is recognised as an important facet of care delivery. Informal caregivers are defined as "persons without formal health care education who are caring for, or helping, a person with functional disabilities, prolonged psychiatric or physical illness, or age-related problems" [4]. Within this chapter, the term "family" 
will be used to encapsulate all persons who have an important relationship with the patient, e.g. relative, partner, friend and neighbour.

Families are an essential component of care, health and wellbeing; quality and safety initiatives recognise the role they play in ensuring high standards of care, and they are allies for quality and safety within the healthcare system [5]. Families often act as the primary caregivers and as advocates for patient who are not able to make decisions for themselves. They are an essential part of the patient care continuum and a fundamental characteristic of holistic care is that nurses must collaborate with others to achieve best practice [6].

There are four keys factors to be considered in relation to patient and family involvement: dignity and respect, information sharing, participation and collaboration. When a patient is admitted to a hospital or other healthcare setting, the reason for admission is often the focus; but, to be holistic, practitioners need to consider a myriad of factors. The reason the patient requires care may not be their own most significant concern. When asked, patients will tell healthcare staff what is worrying them most and it is up to practitioners to really listen and respect their wishes. Nurses spend the most time with the patient and therefore have a central role to play in relaying and advocating for the patient's and family's wishes and concerns, taking into consideration their knowledge, values, beliefs and cultural norms.

Communication is a key ingredient for ensuring timely and appropriate information sharing between healthcare staff and the patient and family. It is known that: "...no matter how knowledgeable a clinician might be, if he or she is not able to open good communication with the patient, he or she may be of no help" [7]; good communication also impacts on patient outcomes and healthcare staff experience. Patients' perceptions of the quality of the healthcare they receive are highly dependent on the quality of their interactions and communication with clinicians and team members [8]. All information shared should be unbiased and relevant; asking the patient or family what it is they need to know or better understand can help avoid frustrations, miscommunication, upset and worry. Practitioners must also communicate the same message to the patient and family to avoid misunderstandings and ensure that they are able to make the most appropriate decision about their care. This two-way dialogue encourages collaboration in achieving good quality decisionmaking and a better ultimate experience for all involved. Practitioners need to adopt a culture of collaboration with families and patients and have a clear programme and structure to ensure this happens at every level of the organisation.

\subsubsection{Assessment}

In the first instance, a comprehensive holistic understanding of the family's role in the care of the patient needs to be achieved and documented. Principal issues for consideration include:

- Who is the legal "next of kin"?

- Do the family want to provide care? 
- Who, if anyone, has previously provided care and what was the care?

- How often is this care required?

- Is it enough to meet the needs of the patient/family?

- Is the environment in which care is provided suitable and adequate?

- What resources or equipment does the patient require, i.e. walking aids?

- What kind of formal care is in place? How often does the patient see their doctor, public health nurse or other carers?

- How have they been managing?

- Have they any worries or perceptions that need to be discussed?

- Are there financial worries for the patient or family in relation to the care required and being provided?

- Is this responsibility causing undue distress?

Families have other priorities such as work and other dependents such as children that need to be considered, and healthcare staff should never assume that the family wish or are able to give care, nor should judgements be made if they prefer not to be involved in direct care. It is important to establish how much support has been, and can be, given by non-family members, the costs associated and how effective this is. Family should be asked if they are aware of any voluntary or communitybased support that can provide resources. Grants and financial help may be available that patients and family can access from social care agencies or voluntary organisations.

The involvement of families in care after discharge requires careful planning and organisation during the discharge planning process, beginning with an open conversation with the patient and their family to ensure that everyone understands what the implications of decisions are. Arrangements should be made for equipment and facilities in the home. Families may need to develop specific care skills, and arrangements need to be in place for this so that they understand issues such as patient limitations and potential continuing progress towards recovery. Should a decision be made that the patient will be discharged permanently or temporarily to residential care, the implications for the family such as social and financial aspects need to be carefully considered with the help of social work practitioners.

\subsubsection{Caregiver Burden}

Voluntary or informal caregiving is a choice and should not be an expectation. Practitioners must take account of the likelihood of caregiver burden, defined as "the physical, emotional and financial responses of a caregiver to the changes and demands caused by providing help to another person with a physical or mental disability" [9]. This is commonly exhibited by families and carers of patients with long-term chronic diseases or acute prolonged episodes of care that can impact physically, psychologically and socially on carers' health and quality of life and, therefore, on the quality of care provided. 
Common features of caregiver burden include tiredness, emotional distress, conflicts, financial difficulties, not meeting the care needs of the patient and changes in the relationship between the patient and carer. A significant cause of these problems is lack of preparation for the caregiver role, poor communication, lack of understanding and appropriate training for the carer, lack of support or perceived support and poor awareness of how to access resources or to navigate health and social care systems.

There is a relationship between informal caregiver wellbeing and the physical and psychological health of the patient, so health and social care services need to ensure that carers are fully supported [10]. This can only be achieved with continued contact and communication. Regular reviews of the care requirements should take place that will enable the carer to be supported. There is a limit to what is reasonable and achievable through informal care and longer-term plans may need to be put in place if care needs are prolonged or carers are not able to cope due to care burden.

\subsubsection{Legal and Ethical Considerations}

The patient is the key decision-maker in their care but, if a patient does not possess the capacity to make decisions about their care, practitioners must act in their best interests to determine who is the correct and legal person to make and inform any decisions made. To do this, the practitioner must understand the terms capacity, advanced healthcare directive (AHD) or living will and co-decision-maker.

Capacity is defined as the person's ability to understand, at the time that a decision is to be made, the nature and consequences of the decision to be made by him or her in the context of the available choices at that time [11]. An expression by a person of his or her will and preferences concerning treatment decisions that may arise if the person subsequently lacks the capacity to make such decisions is known as a living will or an advanced healthcare directive (AHD). The person may appoint someone (known as a co-decision-maker) to jointly or solely make decisions, through a legal, witnessed and documented process, on their behalf should they not possess the capacity to do so.

\subsection{Palliative and End of Life Care}

For some patients, their fragility fracture, especially a hip fracture, may be an event that will hasten the end of life. Some patients, especially those who are already frail, may be unable to survive the physiological stress of the fracture and subsequent surgery. At these times, the principles of palliative care should be applied. The World Health Organization [12] defines palliative care as; "an approach that improves the quality of life of patients and their families facing the problems associated with life-threatening illness, through the prevention and relief of suffering by means of early identification and impeccable assessment and treatment of pain and 
other problems, physical, psychosocial and spiritual". The fundamental aims of palliative care include [12]:

- Providing adequate pain relief and minimising discomfort by providing symptom relief

- Affirming life and regarding dying as a normal process

- Intending neither to hasten nor postpone death

- Integrating the psychological and spiritual aspects of patient care

- Offering a support system to help patients live as actively as possible until death

- Offering to provide a system of support to help the family cope during the patient's illness and death and in their own bereavement

- Working collaboratively as a team to address the needs of patients and their families, including bereavement counselling, if indicated

- Enhancing quality of life and positively influencing the course of illness

- Applicable early in the course of illness, in conjunction with other therapies that are intended to prolong life, such as chemotherapy or radiation therapy, and includes those investigations needed to better understand and manage distressing clinical complications.

It is important to note that surgery for hip fracture may remain the most effective way to manage pain for patients who are reaching the end of life, so the reasons for the decision to undertake surgery must be clearly explained to the patient and family and ethical decision-making employed as discussed earlier.

Palliative care is not limited by time and care should be delivered based on needs as they arise. It can take place in primary care, in acute hospitals and in long-term and hospice care facilities. More people are living longer with more co-morbidities and, unfortunately, the insult of a major fracture such as a hip fracture can see the patient's health decline and ultimately result in end of life. It is estimated that there were approximately 54.6 million deaths worldwide in 2011 and that $9 \%$ of those were due to injuries [13]. Men have a higher risk of mortality after a hip fracture, but women are also at substantial risk of death; this risk exceeds the lifetime risk of death from breast cancer, uterine cancer and ovarian cancer combined. Many patients who survive a hip fracture do not regain their pre-fracture functional level, and almost one third lose their independence [14]. Practitioners must be equipped for, and expect to deliver, end of life and palliative care in the orthopaedic and orthogeriatric setting routinely rather than as an exception.

When considering the philosophy of "end of life care", Dame Cicely Saunders said: "You matter because you are you, and you matter to the end of your life. We will do all we can not only to help you die peacefully, but also to live until you die". Her words reflect the human responsibility to care for others in a humanistic and compassionate way until the end of their life.

There are many responsibilities in end of life care, ranging from communicating with individuals and families about their care and preferences; to observing, discussing and recording any changes in condition and offering compassion and support. A broad range of care skills are needed along with awareness of the values 
which underpin this philosophy of care. When providing end of life care, practitioners should [15]:

- Treat people compassionately

- Listen to people

- Communicate clearly and sensitively

- Identify and meet the communication needs of each individual

- Acknowledge pain and distress and take action

- Recognise when someone may be entering the last few days and hours of life

- Involve people in decisions about their care and respect their wishes

- Keep the person who is reaching the end of their life and those important to them up to date with any changes in condition

- Document a summary of conversations and decisions

- Seek further advice if needed

- Look after yourself and your colleagues and seek support if you need it.

End of life and palliative care are not limited to the acute hospital and can be provided in a range of settings including the community, care homes and hospices. Practitioners need to be attuned to noticing when a person is nearing the end of life or actively dying. How the patient and family are communicated with during this phase of life will depend on the individual patient. As much as possible, this should be patient-led and the nurse should proceed with gentle, honest answers, using a language the person understands. If the patient is uncomfortable, or does not wish to talk about death, it is important to respect their wishes. It is crucial, however, to have sensitive conversations with families and carers to prepare them for impending death.

Good nursing care for those at the end of their life should include physical, emotional and psychological aspects of care along with spiritual support. The process of dying creates multiple emotions and feelings for all involved; the patient, family, carers and the care providers. It can be very stressful and complex. It is helpful to use tools to assist in identifying indicators that someone is approaching their end of life such as the Gold Standards Framework (GSF) [16] and the Palliative Performance Scale 2 (PPS). Nurses play a key role in helping the patient throughout this natural process. The gentle "winding down" at the very end of life can be very peaceful as the body starts to let go, so if the patient is distressed or restless, this can be disrupted.

\subsection{Summary of Main Points for Learning}

- A patient's family and other people meaningful to them are important participants in the care process and need to be recognised as such. Good communication and collaboration with families can be central to achieving high-quality care and good patient outcomes. 
- Much care is provided by family members and other informal carers following discharge. Informal carers need to be educated and supported by practitioners during the discharge planning process to enable them to provide effective care. Potential carer burden must be recognised and support must be provided to prevent it. Informal care cannot continue limitlessly and other more permanent formal care may need to be arranged.

- Fragility fracture, particularly hip fracture, may be a signal of, or hasten, the end of life. Palliative and end of life care are, therefore, important aspects of the care process in both hospital and community settings. Physical, psychological, emotional and spiritual care need to be provided in a sensitive and compassionate manner.

\subsection{Suggested Further Study}

- Find local and national guidance for palliative and end of life care, and use these to identify ways in which care might be improved with respect to these aspects of care.

\subsection{How to Self-Assess Learning}

To identify learning achieved and the need for further study, the following strategies may be helpful:

- Seek feedback from families and carers about their perceptions of the collaborative, family-centred approach employed in your unit.

- Seek advice and mentorship from experts in end of life and palliative care.

- Undertake written reflection about your experiences of end of life and palliative care, and consider whether care could be improved.

- Peer review by colleagues can be used to assess individual progress and practice but should not be too formal. There should be open discussion within the team. Weekly case conferences can identify nurse-focused issues and enable the exchange of expertise. Expertise is conveyed to the various members of the MDT by educational initiatives and by fostering a culture where all the patients' problems are considered.

\section{References}

1. NICE (2011) End of life care for adults: quality standard 13. National Institute for Health and Care Excellence. https://www.nice.org.uk/guidance/qs13

2. Balint $\mathrm{M}$ et al (1969) Training medical students in patient-centered medicine. Compr Psychiatry 10(4):249-258 
3. Shaller D (2007) Patient-centred care: what does it take? Picker Institute and the Commonwealth Fund. http://cgp.pickerinstitute.org/wp-content/uploads/2010/12/shaller.pdf

4. Lethin $\mathrm{C}$ et al (2016) Formal support for informal caregivers to older persons with dementia through the course of the disease: an exploratory, cross-sectional study. BMC Geriatr 16:32

5. Bezold C (2004) The future of patient-centred care: scenarios, visions, and audacious goals. J Altern Complement Med 11(s1):s77-s84

6. Hall C, Ritchie D (2013) What is nursing? Exploring theory and practice, 3rd edn. Sage/ Learning Matters, London

7. Institute for Healthcare Communication (2011) Impact of communication in healthcare. http:// healthcarecomm.org/about-us/impact-of-communication-in-healthcare/

8. Wanzer MB et al (2004) Perceptions of health care providers' communication: relationships between patient-centred communication and satisfaction. Health Commun 16(3):363-384

9. Pearlin LI et al (1990) Caregiving and the stress process; an overview of concepts and their measures. Gerontologist 30:583-594

10. Falaschi P, Eleuteri S (2017) The psychological health of patients and their caregivers. In: Falaschi P, Marsh D (eds) Orthogeriatrics. Switzerland, Springer, pp 201-211

11. Office of the Attorney General. Assisted Decision-Making (Capacity) Act 2015. http://www. irishstatutebook.ie/eli/2015/act/64/enacted/en/html

12. World Health Organization (2014) Global atlas of palliative care at the end of life. www.who. int/nmh/Global_Atlas_of_Palliative_Care.pdf

13. World Health Organization (2013) Global health estimates. Causes of death 2000-2011. www. who.int/healthinfo/global_burden_disease/en

14. Kates SL, Mears SC (2011) A guide to improving the care of patients with fragility fractures. Geriatr Orthop Surg Rehabil 2(1):5-37

15. Royal College of Nursing (2015) RCN end of life care- roles and responsibilities. http:// rcnendoflife.org.uk/my-role/

16. Royal College of General Practitioners (2016) The Gold Standards Framework Proactive Identification Guidance (PIG). https://www.goldstandardsframework.org.uk/cd-content/ uploads/files/PIG/NEW\%20PIG\%20-\%20\%20\%2020.1.17\%20KT\%20vs17.pdf

Open Access This chapter is licensed under the terms of the Creative Commons Attribution 4.0 International License (http://creativecommons.org/licenses/by/4.0/), which permits use, sharing, adaptation, distribution and reproduction in any medium or format, as long as you give appropriate credit to the original author(s) and the source, provide a link to the Creative Commons license and indicate if changes were made.

The images or other third party material in this chapter are included in the chapter's Creative Commons license, unless indicated otherwise in a credit line to the material. If material is not included in the chapter's Creative Commons license and your intended use is not permitted by statutory regulation or exceeds the permitted use, you will need to obtain permission directly from the copyright holder.

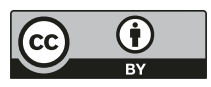

